\title{
Markov operators acting on Polish spaces
}

\author{
by Tomasz Szarek (Katowice)
}

\begin{abstract}
We prove a new sufficient condition for the asymptotic stability of Markov operators acting on measures. This criterion is applied to iterated function systems.

1. Introduction. The purpose of this paper is to present a sufficient condition for asymptotic stability of Markov operators. Our goal is to generalize results of Lasota and Yorke [6] to operators acting on Borel measures defined on Polish spaces. The results of Lasota and Yorke are based on the Prokhorov condition which allows one to construct a stationary distribution. In our case we assume that the metric space is complete and separable (a Polish space) and consequently the space of all probability Borel measures with a suitable metric is a complete metric space.

We will apply our criterion to Markov operators generated by iterated function systems. This class of systems was thoroughly studied because of their close connection with fractals [1], [2], [5], [6], [7], [9].

The organization of the paper is as follows. Section 2 contains some notation from the theory of Markov operators. In Section 3 we give some general conditions for asymptotic stability. These conditions are applied to iterated function systems in Section 4.
\end{abstract}

2. Preliminaries. Let $(X, \varrho)$ be a Polish space, i.e. a separable, complete metric space. This assumption will not be repeated in the statements of theorems. By $\mathcal{M}_{\text {fin }}$ and $\mathcal{M}_{1}$ we denote the sets of Borel measures (nonnegative, $\sigma$-additive) on $X$ such that $\mu(X)<\infty$ and $\mu(X)=1$ respectively. The elements of $\mathcal{M}_{1}$ are called distributions.

We say that $\mu \in \mathcal{M}_{\text {fin }}$ is concentrated on a Borel set $A \subset X$ if $\mu(X \backslash A)$ $=0$. By $\mathcal{M}_{1}^{A}$ we denote the set of all distributions concentrated on the Borel set $A$.

1991 Mathematics Subject Classification: Primary 60J05, 26A18; Secondary 60J20, 39B12.

Key words and phrases: Markov operators, iterated function systems. 
As usual, we denote by $B(X)$ the space of all bounded Borel measurable functions $f: X \rightarrow \mathbb{R}$ and by $C(X)$ the subspace of all bounded continous functions. In both spaces the norm is $\|f\|=\sup _{x \in X}|f(x)|$. For $X$ unbounded, a continuous function $V: X \rightarrow[0, \infty)$ is called a Lyapunov function if

$$
\lim _{\varrho\left(x, x_{0}\right) \rightarrow \infty} V(x)=\infty
$$

for some $x_{0} \in X$.

An operator $P: \mathcal{M}_{\text {fin }} \rightarrow \mathcal{M}_{\text {fin }}$ is called a Markov operator if it satisfies the following two conditions.

(i) positive linearity:

$$
P\left(\lambda_{1} \mu_{1}+\lambda_{2} \mu_{2}\right)=\lambda_{1} P\left(\mu_{1}\right)+\lambda_{2} P\left(\mu_{2}\right)
$$

for $\lambda_{1}, \lambda_{2} \geq 0$ and $\mu_{1}, \mu_{2} \in \mathcal{M}_{\text {fin }}$,

(ii) preservation of the norm:

$$
P \mu(X)=\mu(X) \quad \text { for } \mu \in \mathcal{M}_{\text {fin }} .
$$

It is easy to prove that every Markov operator can be extended to the space of signed measures

$$
\mathcal{M}_{\text {sig }}=\left\{\mu_{1}-\mu_{2}: \mu_{1}, \mu_{2} \in \mathcal{M}_{\text {fin }}\right\} .
$$

Namely for every $\nu \in \mathcal{M}_{\text {sig }}, \nu=\mu_{1}-\mu_{2}$, we set

$$
P \nu=P \mu_{1}-P \mu_{2} \text {. }
$$

To simplify notation we write

$$
\langle f, \nu\rangle=\int_{X} f(x) \nu(d x) \quad \text { for } f \in C(X), \nu \in \mathcal{M}_{\text {sig }} .
$$

An operator $P$ is called a Feller operator if $P$ satisfies (i)-(ii) and there is a linear operator $U: B(X) \rightarrow B(X)$ (dual to $P$ ) such that

$$
\langle U f, \mu\rangle=\langle f, P \mu\rangle \quad \text { for } f \in B(X), \mu \in \mathcal{M}_{\text {fin }}
$$

and

$$
U f \in C(X) \quad \text { for } f \in C(X) .
$$

Assume now that $P$ and $U$ are given. If $f: X \rightarrow \mathbb{R}^{+}\left(\mathbb{R}^{+}=[0, \infty)\right)$ is a Borel measurable function, not necessarily bounded, we may assume that

$$
U f(x)=\lim _{n \rightarrow \infty} U f_{n}(x)
$$

where $\left(f_{n}\right), f_{n} \in B(X)$, is an increasing sequence of functions converging pointwise to $f$. From the Lebesgue monotone convergence theorem it follows that $U f$ satisfies (2.2). 
In the space $\mathcal{M}_{\text {sig }}$ we introduce the Fortet-Mourier norm

$$
\|\nu\|=\sup \{|\langle f, \nu\rangle|: f \in F\}
$$

where $F$ is the subset of $C(X)$ consisting of the functions such that $|f| \leq 1$ and $|f(x)-f(y)| \leq \varrho(x, y)$. It is known that the convergence

$$
\lim _{n \rightarrow \infty}\left\|\mu_{n}-\mu\right\|=0 \quad \text { for } \mu_{n}, \mu \in \mathcal{M}_{1}
$$

is equivalent to the weak convergence of $\left(\mu_{n}\right)$ to $\mu$ (see [4]).

The Markov operator is called nonexpansive if

$$
\left\|P \mu_{1}-P \mu_{2}\right\| \leq\left\|\mu_{1}-\mu_{2}\right\| \quad \text { for } \mu_{1}, \mu_{2} \in \mathcal{M}_{1} .
$$

Let $P$ be a Markov operator. A measure $\mu \in \mathcal{M}_{\text {fin }}$ is called stationary or invariant if $P \mu=\mu$, and $P$ is called asymptotically stable if there exists a stationary distribution $\mu_{\star}$ such that

$$
\lim _{n \rightarrow \infty}\left\|P^{n} \mu-\mu_{\star}\right\|=0 \quad \text { for } \mu \in \mathcal{M}_{1} .
$$

Clearly the distribution $\mu_{\star}$ satisfying (2.6) is unique.

The operator $P$ is called globally concentrating if it has the following property: for every $\varepsilon>0$ and every bounded Borel set $A \subset X$ there exists a bounded Borel set $B \subset X$ and an integer $n_{0}$ such that

$$
P^{n} \mu(B) \geq 1-\varepsilon \quad \text { for } n \geq n_{0}, \mu \in \mathcal{M}_{1}^{A} .
$$

The operator $P$ is called locally concentrating if for every $\varepsilon>0$ there exists $\alpha>0$ such that for every bounded Borel set $A \subset X$ there exists a Borel set $C \subset X$ with $\operatorname{diam} C<\varepsilon$ and an integer $n_{0}$ satisfying

$$
P^{n} \mu(C) \geq \alpha \quad \text { for } n \geq n_{0}, \mu \in \mathcal{M}_{1}^{A} \text {. }
$$

Remark. One can construct a Markov operator which is locally concentrating but is not globally concentrating.

It will be shown in Section 4 that for some IFS $(S, p)$, the corresponding Markov operator is both locally and globally concentrating.

3. Asymptotic stability on Polish spaces. We prove the following criterion of stability.

TheOREM 3.1. Assume that $P$ is a nonexpansive locally and globally concentrating Markov operator. Then $P$ is asymptotically stable.

Proof. First we prove that for every $\mu \in \mathcal{M}_{1}$ the sequence $\left(P^{n} \mu: n \in\right.$ $\mathbb{N}$ ) is convergent. Since the distributions defined on a Polish space with the Fortet-Mourier norm form a complete metric space, it is sufficient to check that the sequence $\left(P^{n} \mu: n \in \mathbb{N}\right)$ satisfies the Cauchy condition. The Cauchy condition can be expressed in the following way: there is $N \in \mathbb{N}$ such that 


$$
\left\|P^{N} \mu_{1}-P^{N} \mu_{2}\right\| \leq \varepsilon
$$

for every $\mu_{1}, \mu_{2} \in\left\{P^{n} \mu: n \in \mathbb{N}\right\}$.

The proof of (3.1) will be done in three steps.

STEP I. We show that for every $\mu \in \mathcal{M}_{1}$ and $\varepsilon>0$ there exists a bounded Borel set $B \subset X$ such that

$$
P^{n} \mu(B) \geq 1-\varepsilon \quad \text { for } n \in \mathbb{N} .
$$

Fix $\varepsilon>0$. In fact, we may take a bounded Borel set $A \subset X$ such that $\mu(A) \geq 1-\varepsilon / 2$. Then $\mu \geq(1-\varepsilon / 2) \mu^{A}$, where $\mu^{A} \in \mathcal{M}_{1}^{A}$ is of the form

$$
\mu^{A}(C)=\frac{\mu(C \cap A)}{\mu(A)} .
$$

By the global concentrating property of $P$ there exists a bounded Borel set $B \subset X$ such that

$$
P^{n} \mu^{A}(B) \geq 1-\varepsilon / 2 \quad \text { for } n \geq n_{0}(A) .
$$

Thus

$$
P^{n} \mu(B) \geq 1-\varepsilon \quad \text { for } n \geq n_{0}(A) .
$$

Enlarging the set $B$ we obtain (3.2).

STEP II. We prove that the Cauchy condition is implied by the following: for every bounded Borel set $A \subset X$ and $\varepsilon>0$ there exists an integer $N$ satisfying

$$
\left\|P^{N} \mu_{1}-P^{N} \mu_{2}\right\| \leq \varepsilon \quad \text { for } \mu_{1}, \mu_{2} \in \mathcal{M}_{1}^{A} .
$$

Fix $\varepsilon>0$. By Step I we can choose a bounded Borel set $A$ such that $\mu_{i}(A) \geq 1-\varepsilon / 4$ for every $\mu_{i} \in\left\{P^{n} \mu: n \in \mathbb{N}\right\}, i=1,2$. Thus

$$
\mu_{i}=\left(1-\frac{\varepsilon}{4}\right) \mu_{i}^{A}+\frac{\varepsilon}{4} \gamma_{i}
$$

where $\mu_{i}^{A}, \gamma_{i} \in \mathcal{M}_{1}$ and are of the form

$$
\mu_{i}^{A}(C)=\frac{\mu_{i}(C \cap A)}{\mu_{i}(A)}, \quad \gamma_{i}(C)=\frac{4}{\varepsilon}\left[\mu_{i}(C)-\left(1-\frac{\varepsilon}{4}\right) \mu_{i}^{A}(C)\right] .
$$

From the nonexpansiveness of $P$ and the inequality $\left\|\gamma_{1}-\gamma_{2}\right\| \leq 2$ it follows that

$$
\begin{aligned}
\left\|P^{N} \mu_{1}-P^{N} \mu_{2}\right\| & \leq\left(1-\frac{\varepsilon}{4}\right)\left\|P^{N} \mu_{1}^{A}-P^{N} \mu_{2}^{A}\right\|+\frac{\varepsilon}{4}\left\|\gamma_{1}-\gamma_{2}\right\| \\
& \leq\left(1-\frac{\varepsilon}{4}\right)\left\|P^{N} \mu_{1}^{A}-P^{N} \mu_{2}^{A}\right\|+\frac{\varepsilon}{2} .
\end{aligned}
$$

Consequently, the Cauchy condition holds. 
SteP III. By Step II it is enough to prove that for every bounded Borel set $A \subset X$ and $\varepsilon>0$ we can choose an integer $N$ such that

$$
\left\|P^{N} \mu_{1}-P^{N} \mu_{2}\right\| \leq \varepsilon \quad \text { for } \mu_{1}, \mu_{2} \in \mathcal{M}_{1}^{A} .
$$

Fix $\varepsilon>0$. Let $\alpha$ be such that (2.8) is satisfied for $\varepsilon / 4$. Let $\delta<\alpha \varepsilon / 4, A_{0}=$ $A$ and $\mu_{i}^{0}=\mu_{i}$ for $i=1,2$. By an induction argument we define a sequence $\left(n_{k}\right)_{k \geq 1}$ of integers, sequences $\left(A_{k}\right)_{k \geq 0},\left(C_{k}\right)_{k \geq 1}$ of bounded Borel sets, $\operatorname{diam} \bar{C}_{k}<\varepsilon / 4$ for $k \in \mathbb{N}$, and sequences $\left(\mu_{i}^{k}\right)_{k \geq 0},\left(\nu_{i}^{k}\right)_{k \geq 1},\left(\lambda_{i}^{k}\right)_{k \geq 1},\left(\tau_{i}^{k}\right)_{k \geq 1}$ of distributions, $i=1,2$, such that $\mu_{i}^{k} \in \mathcal{M}_{1}^{A_{k}}, \nu_{i}^{k} \in \mathcal{M}_{1}^{C_{k}}$ and

$$
\begin{aligned}
P^{n_{k}} \mu_{i}^{k-1} & =(1-\delta) \lambda_{i}^{k}+\delta \tau_{i}^{k}, \\
\lambda_{i}^{k} & =(1-\alpha) \mu_{i}^{k}+\alpha \nu_{i}^{k}
\end{aligned}
$$

and $n_{k}, A_{k}, C_{k}$ depend only on $A_{k-1}$.

Let $A_{0}=A$ and $\mu_{i}^{0}=\mu_{i}$ for $i=1,2$. If $k \geq 1$ is fixed and $\mu_{i}^{k-1}, A_{k-1}$ are given, we choose, according to the global and local concentrating property of $P$, an integer $n_{k}$ and sets $A_{k}, C_{k}$ such that

$$
P^{n_{k}} \mu_{i}^{k-1}\left(A_{k}\right) \geq 1-\delta, \quad P^{n_{k}} \mu_{i}^{k-1}\left(C_{k}\right) \geq \alpha \quad \text { for } i=1,2,
$$

where $n_{k}, A_{k}, C_{k}$ depend only on $A_{k-1}$, and $\operatorname{diam} C_{k}<\varepsilon / 4$. Without loss of generality we assume that $C_{k} \subset A_{k}$. Then we define

$$
\begin{aligned}
\lambda_{i}^{k}(B) & =\frac{P^{n_{k}} \mu_{i}^{k-1}\left(B \cap A_{k}\right)}{P^{n_{k}} \mu_{i}^{k-1}\left(A_{k}\right)}, \\
\tau_{i}^{k}(B) & =\frac{1}{\delta}\left[P^{n_{k}} \mu_{i}^{k-1}(B)-(1-\delta) \lambda_{i}^{k}(B)\right] .
\end{aligned}
$$

Obviously, $\lambda_{i}^{k}\left(C_{k}\right) \geq \alpha$ and we can define

$$
\nu_{i}^{k}(B)=\frac{\lambda_{i}^{k}\left(B \cap C_{k}\right)}{\lambda_{i}^{k}\left(C_{k}\right)}, \quad \mu_{i}^{k}(B)=\frac{1}{1-\alpha}\left[\lambda_{i}^{k}(B)-\alpha \nu_{i}^{k}(B)\right] .
$$

It is clear that $\mu_{i}^{k} \in \mathcal{M}_{1}^{A_{k}}$ and $\nu_{i}^{k} \in \mathcal{M}_{1}^{C_{k}}$. Since $\nu_{i}^{k}\left(X-C_{k}\right)=0$ we have

$$
\begin{aligned}
\left\|\nu_{1}^{k}-\nu_{2}^{k}\right\| & =\sup _{f \in F}\left|\int_{X} f d \nu_{1}^{k}-\int_{X} f d \nu_{2}^{k}\right| \\
& =\sup _{f \in F}\left|\int_{C} f d \nu_{1}^{k}-\int_{C} f d \nu_{2}^{k}\right| \leq \operatorname{diam} C_{k} \leq \frac{\varepsilon}{4} .
\end{aligned}
$$

Setting $a=(1-\delta)(1-\alpha)$ and using equations (3.3), (3.4), it is easy to verify, by an induction argument, that

$$
\begin{aligned}
P^{n_{1}+n_{2}+\ldots+n_{k}} \mu_{i}= & a^{k} \mu_{i}^{k}+(1-\delta) \alpha a^{k-1} \nu_{i}^{k}+\delta a^{k-1} \tau_{i}^{k} \\
& +(1-\delta) \alpha a^{k-2} P^{n_{k}} \nu_{i}^{k-1}+\delta a^{k-2} P^{n_{k}} \tau_{i}^{k-1} \\
& +\ldots+(1-\delta) \alpha P^{n_{2}+\ldots+n_{k}} \nu_{i}^{1}+\delta P^{n_{2}+\ldots+n_{k}} \tau_{i}^{1} .
\end{aligned}
$$


Since $P$ is nonexpansive this implies

$$
\begin{aligned}
&\left\|P^{n_{1}+n_{2}+\ldots+n_{k}}\left(\mu_{1}-\mu_{2}\right)\right\| \\
& \leq a^{k}\left\|\mu_{1}^{k}-\mu_{2}^{k}\right\|+(1-\delta) \alpha a^{k-1}\left\|\nu_{1}^{k}-\nu_{2}^{k}\right\|+\delta a^{k-1}\left\|\tau_{1}^{k}-\tau_{2}^{k}\right\| \\
&+(1-\delta) \alpha a^{k-2}\left\|\nu_{1}^{k-1}-\nu_{2}^{k-1}\right\|+\delta a^{k-2}\left\|\tau_{1}^{k-1}-\tau_{2}^{k-1}\right\| \\
&+\ldots+(1-\delta) \alpha\left\|\nu_{1}^{1}-\nu_{2}^{1}\right\|+\delta\left\|\tau_{1}^{1}-\tau_{2}^{1}\right\| .
\end{aligned}
$$

From this, condition (3.5) and the obvious inequalities $\left\|\mu_{1}^{k}-\mu_{2}^{k}\right\| \leq 2$ and $\left\|\tau_{1}^{1}-\tau_{2}^{1}\right\| \leq 2$, it follows that

$$
\left\|P^{n_{1}+\ldots+n_{k}}\left(\mu_{1}-\mu_{2}\right)\right\| \leq \frac{2}{3} \varepsilon+2 a^{k} .
$$

By Step II the sequence $\left(P^{n} \mu: n \in \mathbb{N}\right)$ satisfies the Cauchy condition. Thus $\left(P^{n} \mu: n \in \mathbb{N}\right)$ converges to some $\mu_{\star} \in \mathcal{M}_{1}$. Obviously $P \mu_{\star}=\mu_{\star}$.

Finally, let $\mu_{1}, \mu_{2} \in \mathcal{M}_{1}$. Fix $\varepsilon>0$. As in Step II we can write

$$
\mu_{i}=\left(1-\frac{\varepsilon}{4}\right) \mu_{i}^{A}+\frac{\varepsilon}{4} \gamma_{i}
$$

where $\mu_{i}^{A} \in \mathcal{M}_{1}^{A}$ for some bounded Borel set $A$ and $\gamma_{i} \in \mathcal{M}_{1}, i=1,2$. We have

$$
\begin{aligned}
\left\|P^{n} \mu_{1}-P^{n} \mu_{2}\right\| & \leq\left(1-\frac{\varepsilon}{4}\right)\left\|P^{n} \mu_{1}^{A}-P^{n} \mu_{2}^{A}\right\|+\frac{\varepsilon}{4}\left\|\gamma_{1}-\gamma_{2}\right\| \\
& \leq\left(1-\frac{\varepsilon}{4}\right)\left\|P^{n} \mu_{1}^{A}-P^{n} \mu_{2}^{A}\right\|+\frac{\varepsilon}{2} .
\end{aligned}
$$

Thus by Step III and nonexpansiveness of $P$ we have for some $N \in \mathbb{N}$,

$$
\left\|P^{n} \mu_{1}-P^{n} \mu_{2}\right\| \leq \varepsilon \quad \text { for } n \geq N .
$$

4. Iterated function systems. In this section we consider some special Markov operators describing the evolution of measures due to the action of a randomly chosen transformation. Assume we are given a sequence of transformations

$$
S_{k}: X \rightarrow X, \quad k=1, \ldots, N
$$

and a probability vector

$$
\left(p_{1}(x), \ldots, p_{N}(x)\right), \quad p_{i}(x) \geq 0, \quad \sum_{i=1}^{N} p_{i}(x)=1,
$$

which depends on the position $x$.

We are going to study the Feller operator [5], [6]

$$
P \mu(A)=\sum_{k=1}^{N} \int_{S_{k}^{-1}(A)} p_{k}(x) \mu(d x) .
$$


Its adjoint operator $U: C(X) \rightarrow C(X)$ is

$$
U f(x)=\sum_{k=1}^{N} p_{k}(x) f\left(S_{k}(x)\right) .
$$

To simplify the language we will say that the Iterated Function System

$$
(S, p)_{N}=\left(S_{1}, \ldots, S_{N}: p_{1}, \ldots, p_{N}\right)
$$

is nonexpansive or asymptotically stable if the Markov operator (4.1) has the corresponding property. We are going to change the metric $\varrho$ in the Polish space $(X, \varrho)$ in such a way that the new space remains a Polish space and the Feller operator $P$ is nonexpansive.

We introduce the class $\Phi$ of functions $\varphi: \mathbb{R}^{+} \rightarrow \mathbb{R}^{+}$satisfying the following conditions:

(i) $\varphi$ is continuous and $\varphi(0)=0$;

(ii) $\varphi$ is nondecreasing and concave, i.e. $\frac{1}{2} \varphi\left(t_{1}\right)+\frac{1}{2} \varphi\left(t_{2}\right) \leq \varphi\left(\frac{t_{1}+t_{2}}{2}\right)$ for $t_{1}, t_{2} \in \mathbb{R}^{+}$

(iii) $\varphi(x)>0$ for $x>0$ and $\lim _{x \rightarrow \infty} \varphi(x)=\infty$.

We denote by $\Phi_{0}$ the family of functions satisfying (i)-(ii). It is easy to see that for every $\varphi \in \Phi$ the function

$$
\varrho_{\varphi}(x, y)=\varphi(\varrho(x, y)) \quad \text { for } x, y \in X
$$

is again a metric on $X$ and $\left(X, \varrho_{\varphi}\right)$ is a Polish space.

In our considerations an important role is played by the inequality

$$
\omega(t)+\varphi(r(t)) \leq \varphi(t) \quad \text { for } t \geq 0 .
$$

Lasota and Yorke [6] discussed three special cases for which inequality (4.2) has solutions belonging to $\Phi$.

Case I: Dini condition. Assume that $\omega \in \Phi_{0}$ satisfies the Dini condition, i.e.

$$
\int_{0}^{\varepsilon} \frac{\omega(t)}{t} d t<\infty \quad \text { for some } \varepsilon>0
$$

and $r(t)=c t, 0 \leq c<1$.

CASE II: Hölder condition. Assume that $\omega \in \Phi_{0}$,

$$
\omega(t) \leq a t^{\beta},
$$

where $a>0$ and $\beta>0$ are constants, $r \in \Phi_{0}, r(t)<t$ and

$$
0 \leq r(t) \leq t-t^{\alpha+1} b \quad \text { for } 0 \leq t \leq \varepsilon,
$$

where $\alpha>0, b>0$ and $\varepsilon>0$ are constants. 
CASe III: Lipschitz condition. Assume that $\omega \in \Phi_{0}$,

$$
\omega(t) \leq a t,
$$

where $a>0$ is a constant, and $r \in \Phi_{0}$ satisfies the conditions

$$
\begin{array}{ll}
0 \leq r(t)<t & \text { for } t>0, \\
\int_{0}^{\varepsilon} \frac{t d t}{t-r(t)}<\infty & \text { for some } \varepsilon>0 .
\end{array}
$$

In Cases I-III the iterates $r^{n}$ of the function $r$ converge to 0 and the function

$$
\varphi(t)=t+\sum_{n=0}^{\infty} \omega\left(r^{n}(t)\right)
$$

is a solution of the inequality (4.2) from $\Phi$.

Now assume that

$$
\begin{gathered}
\sum_{k=1}^{N}\left|p_{k}(x)-p_{k}(y)\right| \leq \omega(\varrho(x, y)), \\
\sum_{k=1}^{N} p_{k}(x) \varrho\left(S_{k}(x), S_{k}(y)\right) \leq r(\varrho(x, y)) .
\end{gathered}
$$

We have

$$
\left\|P \mu_{1}-P \mu_{2}\right\|_{\varphi}:=\sup _{F_{\varphi}}\left|\left\langle f, P \bar{\mu}_{1}-P \bar{\mu}_{2}\right\rangle\right|=\sup _{F_{\varphi}}\left|\left\langle U f, \mu_{1}-\mu_{2}\right\rangle\right|,
$$

where $F_{\varphi}$ is the set of all functions on $X$ such that $|f| \leq 1$ and

$$
|f(x)-f(y)| \leq \varrho_{\varphi}(x, y) .
$$

The operator $P$ is nonexpansive with respect to $\varrho_{\varphi}$ if $U f \in F_{\varphi}$ for $f \in F_{\varphi}$. Of course $|U f| \leq 1$, so we have to prove that

$$
|U f(x)-U f(y)| \leq \varrho_{\varphi}(x, y) .
$$

We have

$$
\begin{aligned}
|U f(x)-U f(y)| & =\left|\sum_{k=1}^{N} p_{k}(x) f\left(S_{k}(x)\right)-\sum_{k=1}^{N} p_{k}(y) f\left(S_{k}(y)\right)\right| \\
& \leq \sum_{k=1}^{N}\left|p_{k}(x)-p_{k}(y)\right|+\sum_{k=1}^{N} p_{k}(y)\left|f\left(S_{k}(x)\right)-f\left(S_{k}(y)\right)\right| \\
& \leq \omega(\varrho(x, y))+\sum_{k=1}^{N} p_{k}(y) \varphi\left(\varrho\left(S_{k}(x), S_{k}(y)\right)\right.
\end{aligned}
$$




$$
\begin{aligned}
& \leq \omega(\varrho(x, y))+\varphi\left(\sum_{k=1}^{N} p_{k}(y) \varrho\left(S_{k}(x), S_{k}(y)\right)\right) \\
& =\omega(\varrho(x, y))+\varphi(r(\varrho(x, y))) .
\end{aligned}
$$

If the pair $(\omega, r)$ satisfies the conditions formulated in one of Cases I-III and $\varphi$ is a solution of the inequality (4.2), then (4.5) is satisfied.

Now we prove the following lemma.

Lemma 4.1. Let $P$ be a Feller operator and $U$ its dual. Assume that there is a Lyapunov function $V$ such that $V$ is bounded on bounded sets and

$$
U V(x) \leq a V(x)+b \quad \text { for } x \in X
$$

where $a, b$ are nonnegative constants and $a<1$. Then $P$ is globally concentrating.

Pr o of. From (4.6) it follows that

$$
U^{n} V(x) \leq a^{n} V(x)+\frac{b}{1-a} .
$$

Fix $\varepsilon>0$. Let $A$ be a bounded Borel set and $\mu \in \mathcal{M}_{1}$. Let

$$
B=\{x: V(x) \leq q\},
$$

where $q>2 b /((1-a) \varepsilon)$. From the Chebyshev inequality we obtain

$$
\begin{aligned}
P^{n} \mu(B) & \geq 1-\frac{1}{q} \int_{X} V(x) P^{n} \mu(d x)=1-\frac{1}{q} \int_{X} U^{n} V(x) d \mu \\
& \geq 1-\frac{1}{q}\left(a^{n} \int_{X} V(x) d \mu+\frac{b}{1-a}\right) \geq 1-\frac{\varepsilon}{2}-\frac{a^{n}}{q} \int_{X} V(x) d \mu \\
& \geq 1-\frac{\varepsilon}{2}-\frac{a^{n}}{q} \sup _{x \in A} V(x) .
\end{aligned}
$$

Consequently, there exists an integer $n_{0}$ such that

$$
P^{n} \mu(B) \geq 1-\varepsilon \quad \text { for } n \geq n_{0}, \mu \in \mathcal{M}_{1}^{A} .
$$

Now we prove the main theorem of this paper.

Theorem 4.2. Assume that the pair $(\omega, r)$ defined by (4.3), (4.4) satisfies the conditions of one of Cases I-III. Moreover, assume that

$$
\inf _{x \in X} p_{k}(x)>0 \quad \text { for } k=1, \ldots, N \text {. }
$$

Finally, suppose that for every bounded Borel set $B \subset X$ and every $\varepsilon>0$ there exists an integer $n_{0}$ and a sequence $\left(i_{1}, \ldots, i_{n_{0}}\right), i_{1}, \ldots, i_{n_{0}} \in\{1, \ldots$ $\ldots, N\}$, such that

$$
\operatorname{diam}\left(S_{i_{n_{0}}} \circ \ldots \circ S_{i_{1}}(B)\right)<\varepsilon .
$$

Then the system $(S, p)_{N}$ is asymptotically stable. 
Proof. We show that the Markov operator corresponding to $(S, p)_{N}$ satisfies the assumptions of Lemma 4.1.

It is easy to check that

$$
\sum_{k=1}^{N} p_{k}(x) \varrho\left(S_{k}(x), x_{0}\right) \leq r(1) \varrho\left(x, x_{0}\right)+r(1)+\max _{1 \leq k \leq N} \varrho\left(S_{k}\left(x_{0}\right), x_{0}\right) .
$$

Thus the assumptions of Lemma 4.1 are satisfied with $V(x)=\varrho\left(x, x_{0}\right), a=$ $r(1)<1$ and $b=r(1)+\max _{1 \leq k \leq N} \varrho\left(S_{k}\left(x_{0}\right), x_{0}\right)$. From Lemma 4.1, it follows that $P$ is globally concentrating. Since the conditions required in one of Cases I-III are satisfied, there is a solution $\varphi \in \Phi$ of (4.2) and the system $(S, p)_{N}$ is nonexpansive with respect to the metric $\varrho_{\varphi}=\varphi \circ \varrho$.

By an induction argument it is easy to verify that

$$
\begin{aligned}
& P^{n} \mu(A)=\left\langle\mathbf{1}_{A}, P^{n} \mu\right\rangle=\left\langle U^{n} \mathbf{1}_{A}, \mu\right\rangle \\
& \quad=\sum_{k_{1}, \ldots, k_{n}} \int_{X} p_{k_{1}}(x) \ldots p_{k_{n}}\left(S_{k_{n-1}, \ldots, k_{1}}(x)\right) \mathbf{1}_{A}\left(S_{k_{n}, \ldots, k_{1}}(x)\right) d \mu(x),
\end{aligned}
$$

where $S_{k_{n}, \ldots, k_{1}}=S_{k_{n}} \circ \ldots \circ S_{k_{1}}$.

We end the proof when we show that the operator $P$ is locally concentrating. Following the proof of Lemma 4.1 it is easy to show that for the set

$$
B=\{x: V(x) \leq 2 b /(1-a)\},
$$

for every bounded Borel set $A$ there exists an integer $n_{0}$ such that

$$
P^{n} \mu(B) \geq 1 / 4 \quad \text { for } n \geq n_{0}, \mu \in \mathcal{M}_{1}^{A} \text {. }
$$

Fix $\varepsilon>0$. Using (4.8) we can take $n_{1} \in \mathbb{N}$ and a sequence $\left(i_{1}, \ldots, i_{n_{1}}\right)$, $i_{1}, \ldots, i_{n_{1}} \in\{1, \ldots, N\}$, such that

$$
\varphi\left(\operatorname{diam}\left(S_{i_{1}} \circ \ldots \circ S_{i_{n_{1}}}(B)\right)\right) \leq \varepsilon .
$$

Let $C=S_{i_{1}} \circ \ldots \circ S_{i_{n_{1}}}(B)$. We have

$$
\operatorname{diam}_{\varrho \varphi}(C)=\varphi(\operatorname{diam} C) \leq \varepsilon .
$$

Fix a bounded Borel set $A \subset X$. There exists an integer $n_{0}$ such that

$$
P^{n} \mu(B) \geq 1 / 4 \quad \text { for } n \geq n_{0}, \mu \in \mathcal{M}_{1}^{A} \text {. }
$$

Thus for $n \geq n_{1}+n_{0}$ using (4.9) we have

$$
\begin{aligned}
& P^{n} \mu(\bar{C})=P^{n_{1}}\left(P^{n-n_{1}} \mu\right)(\bar{C}) \\
& \quad=\sum_{k_{1}, \ldots, k_{n_{1}}} \int_{X} p_{k_{1}}(x) \ldots p_{k_{n_{1}}}\left(S_{k_{n_{1}-1}, \ldots, k_{1}}(x)\right) \mathbf{1}_{\bar{C}}\left(S_{k_{n_{1}}, \ldots, k_{1}}(x)\right) d P^{n-n_{1}} \mu(x)
\end{aligned}
$$




$$
\begin{aligned}
& \geq \int_{X} p_{i_{1}}(x) \ldots p_{i_{n_{1}}}\left(S_{i_{n_{1}-1}, \ldots, i_{1}}(x)\right) \mathbf{1}_{\bar{C}}\left(S_{i_{n_{1}}, \ldots, i_{1}}(x)\right) d P^{n-n_{1}} \mu(x) \\
& \geq \inf _{x \in X} p_{i_{1}}(x) \ldots \inf _{x \in X} p_{i_{n_{1}}}(x) P^{n-n_{1}} \mu(B) \\
& \geq \inf _{x \in X} p_{i_{1}}(x) \ldots \inf _{x \in X} p_{i_{n_{1}}}(x) \cdot \frac{1}{4} .
\end{aligned}
$$

Thus $P$ is locally concentrating. According to Theorem 3.1 the proof is complete.

EXAMPLE. It is interesting to compare our results with a theorem of $\mathrm{K}$. Eoskot and R. Rudnicki. Their result assures the asymptotic stability of $(S, p)_{N}$ under the following conditions:

(i) $(X, \varrho)$ is a Polish space,

(ii) $p_{k}: X \rightarrow \mathbb{R}, k=1, \ldots, N$, are constant,

(iii) $S_{k}: X \rightarrow X, k=1, \ldots, N$, are Lipschitzian,

(iv) $\sum_{k=1}^{N} p_{k} L_{k}<1$, where $L_{k}$ is the Lipschitz constant of $S_{k}$.

It is easy to check that the assumptions formulated in Theorem 4.2 are satisfied. The asymptotic stability of this system follows from our Theorem.

\section{References}

[1] M. F. Barnsley, Fractals Everywhere, Academic Press, New York, 1988.

[2] M. F. Barnsley, V. Ervin, D. Hardin and J. Lancaster, Solution of an inverse problem for fractals and other sets, Proc. Nat. Acad. Sci. U.S.A. 83 (1986), 19751977.

[3] P. Billingsley, Convergence of Probability Measures, Wiley, New York, 1968.

[4] R. M. Dudley, Probabilities and Metrics, Aarhus Universitet, 1976.

[5] A. Lasota, From fractals to stochastic differential equations, to appear.

[6] A. Lasota and J. A. Yorke, Lower bound technique for Markov operators and iterated function systems, Random Comput. Dynam. 2 (1994), 41-77.

[7] K. Loskot and R. Rudnicki, Limit theorems for stochastically perturbed dynamical systems, J. Appl. Probab. 32 (1995), 459-469.

[8] K. Parthasarathy, Probability Measures on Metric Spaces, Academic Press, New York, 1967.

[9] T. Szarek, Iterated function systems depending on previous transformations, to appear.

Institute of Mathematics

Polish Academy of Sciences

Staromiejska 8/6

40-013 Katowice, Poland

E-mail: szarek@gate.math.us.edu.pl 